\title{
Studying Cavitation Enhanced Therapy
}

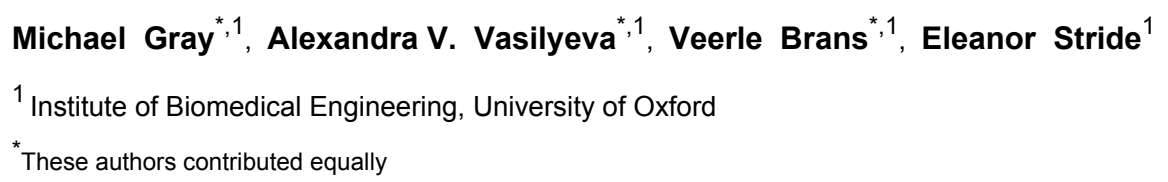

\section{Corresponding Authors}

Michael Gray

michael.gray@eng.ox.ac.uk

Eleanor Stride

eleanor.stride@eng.ox.ac.uk

\section{Citation}

Gray, M., Vasilyeva, A.V., Brans, V., Stride, E. Studying Cavitation Enhanced Therapy. J. Vis. Exp. (170), e61989, doi:10.3791/61989 (2021).

\section{Date Published}

April 9, 2021

\section{DOI}

$10.3791 / 61989$

URL

jove.com/video/61989

\section{Abstract}

Interest in the therapeutic applications of ultrasound is significant and growing, with potential clinical targets ranging from cancer to Alzheimer's disease. Cavitation - the formation and subsequent motion of bubbles within an ultrasound field represents a key phenomenon underpinning many of these treatments. There remains, however, considerable uncertainty regarding the detailed mechanisms of action by which cavitation promotes therapeutic effects and there is a need to develop reliable monitoring techniques that can be implemented clinically. In particular, there is significant variation between studies in the exposure parameters reported as successfully delivering therapeutic effects and the corresponding acoustic emissions. The aim of this paper is to provide design guidelines and an experimental protocol using widely available components for performing studies of cavitation-mediated bioeffects, and include real-time acoustic monitoring. It is hoped that the protocol will enable more widespread incorporation of acoustic monitoring into therapeutic ultrasound experiments and facilitate easier comparison across studies of exposure conditions and their correlation to relevant bio-effects.

\section{Introduction}

Ultrasound (US) has been used widely as a diagnostic imaging technique because of its safe and non-invasive nature, its ease of implementation at a patient's bedside, and its cost effectiveness ${ }^{1}$. Next to its diagnostic and monitoring capabilities, US has considerable potential for therapeutic applications. Early work explored its use in thrombolysis, DNA transfection, and drug delivery $2,3,4$ and therapeutic US now represents a very active area of research, with applications including tumor treatment ${ }^{5,6,7}$, immunotherapy ${ }^{8,9}$, bloodbrain barrier (BBB) disruption ${ }^{10,11,12}$, thrombolysis ${ }^{13,14,15}$, and bacterial infection treatment ${ }^{16,17}$. A key phenomenon underpinning these applications is cavitation: the nucleation, growth, and oscillation of gaseous cavities due to changes in fluid pressure ${ }^{18,19}$

There is a range of mechanisms by which cavitation produces biological effects. For example, the highly nonlinear nature of bubble oscillations under the influence of an applied US field can generate microstreaming in the surrounding liquid that can both enhance drug convection ${ }^{20}$ and exert shear 
stresses on the tissue in the vicinity of the bubbles. This is particularly prevalent when bubbles are in the vicinity of a boundary, causing bubbles to oscillate non-spherically, and may potentially promote drug uptake through shearinduced permeabilization 21,22,23,24. At higher pressures, larger amplitude oscillations and rapid bubble collapse are observed, imparting direct mechanical stress ${ }^{25}$ and frequently generating shock waves, and consequent large pressure gradients that can disrupt and permeabilize tissues 26,27 . The collapse of bubbles near a surface can also result in the formation of high-velocity liquid microjets $^{28,29,30}$. These microjets can penetrate tissue, potentially creating pores or inducing secondary stress waves $^{31,32}$. The permeabilization of biological membranes at both the tissue and cellular levels is variously referred to as sonophoresis, used primarily in the context of US-induced enhancement in skin permeability 33,34 , and sonoporation, used mainly to describe the reversible permeabilization of the cellular membrane due to formation of membrane pores ${ }^{35,36}$.

Viscous absorption in the liquid immediately surrounding the oscillating bubble can produce a substantial heating effect ${ }^{37}$. Moreover, the highly non-linear oscillations produce acoustic radiation at frequencies higher than the driving US field. This leads to increased absorption in the surrounding tissue and further heating ${ }^{38}$. Bubble collapse may also be accompanied by chemical effects due to the transient high temperatures and pressures in the bubble core, such as generation of highly reactive species and electromagnetic radiation, known as sonoluminescence ${ }^{32}$. These effects have been investigated to assess potential damage and/or activation of relevant cellular pathways for delivery ${ }^{39}$ and exploited in local activation of light-sensitive drugs in an approach known as sonodynamic therapy $40,41,42,43$.
Many US-mediated bioeffects may be initiated solely through control of US field parameters (pressure amplitude, frequency, pulse length and repetition frequency, and duration of exposure), but reliably generating cavitation in biological tissue often requires high input energies and hence carries an elevated risk of damage. Introduction of exogenous or artificial cavitation nuclei may substantially reduce the input energy required to produce the broad range of effects discussed above and further introduces additional effects that may not be possible with US alone. Cavitation nuclei

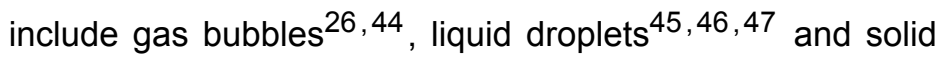
particles $^{48,49,50}$, with nanoscale cavitation nuclei being an emergent area of investigation for their benefits in terms of prolonged circulation time, improved extravasation and prolonged cavitation activity $49,51,52,53$.

The most commonly used nuclei are gas microbubbles (MBs), originally used as contrast agents in diagnostic imaging. They are typically 1-2 micrometers in diameter and contain a core of a high-molecular-weight gas with low aqueous solubility in the surrounding medium. The core is surrounded by a protective lipid, protein, or polymer shell most commonly consisting of phospholipids ${ }^{54}$. When exposed to a US field, the compressibility of the MBs causes them to undergo volumetric oscillations, consequently producing strong acoustic scattering, which is responsible for the success of MBs as a contrast agent. As mentioned, these oscillations also lead to the aforementioned mechanical, thermal, and chemical effects that can be harnessed in therapeutic applications. The MB coating process also offers a mechanism for encapsulating drugs within the MB structure and for attaching drugs and/or targeting species to the MB surface. This technique facilitates the triggered release of drugs to reduce systemic toxicity ${ }^{55}$. It has also recently been shown that material from the MB surface may be transferred 
to biological structures, enhancing drug delivery through so called "sonoprinting" $56,57,58$.

Monitoring of US-mediated cavitation activity can provide insights into the resultant biological effects both in vitro and in vivo and potentially allows for the tuning and optimization of these effects. The two most widely applied methods for monitoring cavitation activity are i) optical, which use ultra high speed video microscopy and are generally not feasible in vivo; and ii) acoustic, which record the re-radiated sound fields produced by oscillating and/or collapsing bubbles. Both the amplitude and frequency components of the acoustic signal contain information on bubble behavior. Low concentrations of bubbles at low incident US amplitudes have been shown to produce predominantly harmonic emissions (integer multiples of the driving frequency) ${ }^{59}$. As driving pressures increase, the bubble emission spectrum may also contain fractional components known as subharmonics and ultraharmonics $^{60}$ that indicate stronger nonlinear behavior, as well as broadband noise, which is indicative of inertial cavitation. Integer harmonics are a primary indicator of bubble oscillation but can also be caused by non-linearities anywhere in an experimental system, e.g., due to nonlinear propagation. By contrast, fractional harmonics and broadband noise are very strongly correlated with bubble dynamics.

The relationship between bubble behavior and the detected acoustic emissions may be complicated by factors including the incident US field, the nucleation environment, and the characteristics of the detection pathway ${ }^{60}$. Nevertheless, important information about bubble behavior and their interactions with cells can be gained by discerning trends in frequency and energy in the acoustic spectrum. These data can also provide valuable information that can be used to form the basis for clinical treatment monitoring techniques. To fully exploit this information, the development of robust, translatable, and reproducible experimental methods is required.

Currently there is substantial variation in reported protocols for designing systems and conducting studies to support development of cavitation-assisted therapies. In terms of the apparatus, a range of design approaches has been undertaken. Several groups have made use of parallel-plate chambers $56,61,62,63$, either custom built or commercially available (e.g., OptiCell, ThermoFisher Scientific). Hu et al. (2013) developed a cell chamber coupled with an US sonication module and real-time confocal imaging ${ }^{64}$, Carugo et al. (2015) used a system comprising a commercially available cell culture dish with a custom-made PDMS lid to allow for submersion in a water bath during US exposure ${ }^{65}$, and Pereno et al. (2018) used a device consisting of layered acoustofluidic resonators that allow for simultaneous optical and acoustic characterization of bubble dynamics and bubble-cell interactions ${ }^{66}$. The use of custom-fabricated and application-specific designs complicates characterization of the US field and other environmental exposure conditions, making cross study comparisons challenging. For example, there is considerable variation in the US parameters identified for achieving successful sonoporation, which include center frequencies ranging from 0.02 to $15 \mathrm{MHz}$, duty cycles varying from $1 \%$ to continuous wave, and rarefactional pressures ranging from 0.1 to $20 \mathrm{MPa}^{23,64,67,68,69,70}$ (Table 1). There is similarly considerable variation in the spectral components (harmonics, sub-harmonics etc.) that have been identified as being associated with particular bioeffects.

The aim of this work, therefore, is to provide an easily reproducible system design and implementation framework 
for the in vitro study of cavitation-induced cellular bioeffects with the specific inclusion of a cavitation monitoring capability.

\section{Protocol}

\section{System Design Principles}

NOTE: This section presents the design principles used to create systems for US exposure and cavitation monitoring. These principles are illustrated with two existing systems for acoustic transfection (SAT) (shown in Figure 1). Each system consists of a cell exposure compartment, an US source, and a single element transducer functioning as a passive cavitation detector (PCD), all of which are integrated into a benchtop test chamber. These designs build upon the prior system development described in Carugo et al. $(2015)^{65}$.

1. Maximize ease of use.

1. Make the cell exposure compartment compatible with existing culture techniques and imaging systems by using existing commercial cell culture devices as seeding/growth substrates.

1. For SAT2, use a culture dish (35 $\mathrm{mm}$ diameter, of which a $21 \mathrm{~mm}$ diameter area is observable, see Table of Materials).

2. For SAT3, use a Transwell insert $(6.5 \mathrm{~mm}$ diameter, see Table of Materials). The Transwells have a permeable membrane and hence need to be placed in cell media rather than water.

2. Enable rapid loading and sealing of the cell exposure compartment.

1. Form the SAT2 cell exposure compartment by press fitting a flexible polymer lid over the culture dish
(Carugo et al. $2015^{65}$ ). As seen in Figure 1C, the lid has a pair of $1.2 \mathrm{~mm}$ diameter holes that allow filling the compartment with an $18 \mathrm{G}$ blunt needle syringe. After filling, seal these filling ports with short plastic rods (Table of Materials).

2. Fill the SAT3 compartment by syringe or pipette and seal by press fitting a rubber stopper/bung.

3. Enable rapid loading of the sealed cell exposure compartment into the test chamber. Holders for the cell exposure compartments were built into the chamber lids, where a light press-fit is sufficient to ensure proper alignment. With the systems shown in Figure 1, the time for sample changes can be as short as 20 seconds when multiple cell exposure compartments have been prepared in advance.

4. Minimize the chamber internal volume so that the system is portable and the amount of required water/ media can be minimized. Doing so also accelerates recovery from accidental spills or leaks of cavitation agents out of the cell exposure compartment.

NOTE: The SAT2 internal volume is approximately 0.8 L. The SAT3 internal volume is approximately 7.6 $\mathrm{L}$ - made larger to accommodate easy loading and change of source transducer or its configuration. An internal chamber of $0.3 \mathrm{~L}$ was added to minimize the disposable volume and to allow biologically relevant fluids other than the tank fill water (e.g., cell culture media) to be used. The internal chamber bottom is made from $30 \mu \mathrm{m}$ thick mylar sheet to allow maximum acoustic transmission.

5. Make the chamber and the internal components out of optically clear materials when possible, so that 
any problems (e.g., leaks, entrapped macrobubbles) can be quickly observed and remedied.

3. Maximize acoustic transmissibility of the exposure compartment.

1. Maximize transmissibility through the choices of compartment wall materials and thicknesses. Under the assumption that the liquids on either side of a wall are essentially the same (e.g., water), the magnitude of the normal incidence pressure transmission coefficient ${ }^{71}$ is:

$|T|=\left(1+\left(\gamma \sin \left(\frac{2 \pi L}{\lambda}\right)\right)^{2}\right)^{-\frac{1}{2}}$, where $\lambda$ is the wavelength in the wall of thickness $L, \gamma=0.5 \cdot\left|z_{L} / z_{o}-z_{o} / z_{L}\right|$, and $z_{L}$ and $z_{O}$ are the characteristic impedances (products of density and sound speed) for the wall material and liquid, respectively. $T=1$ indicates perfect transmission.

2. For broad spectrum monitoring of cavitation (e.g., 1-8 MHz), most laboratory polymers (e.g., PDMS, PTFE, polystyrene) will alter the transmitted pressure by no more than $10 \%$ if the thickness of material is less than $1 / 10^{\text {th }}$ of a wavelength in the material. This condition can be difficult to meet with standard supplies at high frequencies (e.g., \#1.5 coverslip at $8 \mathrm{MHz}$ ), so it is good practice to predict or directly calibrate the transmission frequency response.

3. For narrow band transmission of the US source signal into the cell exposure compartment, allow a thicker layer if it is approximately an integer multiple of a half wavelength in the layer material. For example, the PDMS lid in SAT2 is used at a thickness of $2.0 \mathrm{~mm}$ ( $\sim 2$ wavelength at $1 \mathrm{MHz}$, CPDMS $\sim 1000 \mathrm{~m} / \mathrm{s}$ ).

4. Maximize the exposure region through selection of source and cell environment.

1. To maximize the number of exposed cells, make the area of cell attachment as broad as possible while maintaining compatibility with available culturing and imaging equipment.

2. Use an US source with a field that spans the cell attachment area with minimal spatial variability by using the pre-focal region of a large focused source (SAT2) or a focused or lensed source with a main lobe width that matches the diameter of the cell attachment area (SAT3). See the Table of Materials for specific sources.

3. Minimize the field complexity introduced by the cell compartment holder by mechanically supporting the cell compartment well away from the strongest part of the incident field, minimizing the scattering cross section of the holder or placing absorbing material on the holder. Examples are shown in Figure 1A and $1 D$.

5. Ensure repeatable exposure conditions.

1. Terminate the acoustic field in a fixed boundary to eliminate variability that can arise from air-water interfaces in partially filled chambers. In SAT2 and 3 , this is accomplished by installing an acoustic absorber (see Table of Materials) on the chamber lid with a further benefit of reducing the field complexity that may arise from boundary reflections.

2. Monitor and record the source drive voltage at the amplifier output/source input so that minor variability or major malfunction can be detected quickly. Use 
a voltage probe or other device that is safe to use over the drive voltage range of interest. Periodically check the calibration of the voltage probe using a well-known source such as a waveform generator.

3. Control, monitor, and record the temperature of the chamber and its contents. Responses of cells, transducers, and propagation medium may all be temperature sensitive. In SAT2, monitoring and control are accomplished with a pair of circulation ports connected to a water conditioning system, whilst SAT3 employs an aquarium heater (not shown). Set the water temperature as needed to mimic the relevant physiological conditions for the therapeutic application.

NOTE: internal temperatures may change both as a result of external factors and from US-generated heating of the transducer and the medium.

4. Carefully degas the chamber liquid(s) to minimize the likelihood of unintended cavitation and/ or scattering from pre-existing bubbles in the propagation path.

NOTE: if degassing is not carried out, e.g. due to the negative impact on cells, then there will be an enhanced background level of bubble activity, for which suitable.

6. Calibrate the fully assembled system.

1. Include a means of measuring the pressure field incident upon the exposed cells when all system components are in place, including the cell exposure compartment. In SAT2 and 3, this is accomplished with an opening in the chamber lid through which a needle or fiber optic hydrophone could be inserted without disturbing the field to be measured. Make the measurements as close as possible to where the cells are located.

2. Choose a hydrophone with a sensitive radius ( $\left.a_{r c v}\right)$ is small enough that it will not misreport the pressure field being measured. The acceptable size is a function of source frequency $(f)$ and radius $\left(a_{s r c}\right)$ as well as the distance between source and the field scan $\left(z_{r c v}\right)$. A general criterion for hydrophone size selection is: $\frac{4 \pi f}{c} \frac{a_{s r c} a_{r c v}}{z_{r c v}}<1$, leading to: $a_{r c v}<\frac{c}{4 \pi f} \frac{z_{r c v}}{a_{s r c}}$, where $c$ is the sound speed ${ }^{72}$.

3. Ensure that the hydrophone is calibrated under the conditions used in system characterization, including the temperature as specified in section 1.6. Specifically, if the hydrophone is held at an angle with respect to the scan plane, the hydrophone must be calibrated at that angle, as directivity effects may differ significantly from those expected based purely on geometry. The change in hydrophone sensitivity with respect to temperature should be available from the manufacturer.

4. Scan the entire region in which cells may be exposed. To capture an appropriate level of field detail, use a scan spacing no coarser than $1 / 5^{\text {th }}$ of a wavelength at the highest frequency of interest. If unexpected field complexity is observed, consider using short burst signals (e.g., 1-3 cycles) to allow identification and quantification of direct and scattered field contributions.

7. Incorporate a cavitation monitoring capability.

1. Determine the monitoring transducer type and placement as part of the design of the overall system, rather than as a retrofit. In practice, this 
leads to a system that is maximally compact without sacrificing the ability to reliably align the critical system components.

2. Place a cavitation monitoring device in the system in such a way that it can be repeatably positioned with minimal added setup time or disturbance to workflow. In SAT2, this is accomplished with a single element piezoelectric transducer acting as a PCD fitted into the chamber lid, whilst SAT3 integrates the PCD into the source base using a $90^{\circ}$ reflector.

3. Select the PCD shape according to the objectives of the experiments. In Figure 2, calculations of the halfamplitude contours of unfocused (left) and focused (right) devices show the profound differences in spatial sensitivity with respect to frequency. The unfocused device is better suited for large volume monitoring with modest spatial variation with respect to frequency, while the focused device is better suited for more radially compact measurements at the frequencies of interest.

4. Select the PCD center frequency and bandwidth to fit the needs of the experiment. The center frequency is typically chosen to be at least five times that of the US source in order to minimize sensitivity to direct source emissions. Bandwidth is typically maximized in order to observe a broad range of bubble behaviors (harmonic and broadband noise).

5. Select conditioning, recording, and processing methods to allow analysis of cavitation data, as described in the next section.

\section{Instrumentation and Processing for Cavitation Monitoring}

NOTE: This section presents the signal flow components and functions recommended for collection of cavitation monitoring data, and the data processing that leads to qualitative and quantitative assessments of cavitation activity.

1. Instrumentation (See also Figure 3).

1. Unless the application calls for a customized device, select a PCD from the broad range of commercially available single element transducers, typically marketed for non-destructive testing of submerged targets. These suppliers also have cabling and accessories (e.g., the mirror reflector in SAT3).

2. Minimize PCD response to the US source. This may be done both through selection of the PCD (center frequency and bandwidth) and by using a notch or high pass filter. The latter is implementable either as a standalone module or as part of a signal conditioning device.

3. Use a digitizer with a large dynamic range (at least 12 bits) to capture as much data as possible with minimal likelihood of high signal clipping and maximal signal to noise ratio (SNR) of the smallest signals. When comparing devices, review the specifications for signal to noise and distortion and/or effective number of bits, as these are more complete descriptions of attainable dynamic range. Also consider whether the size of the memory buffer is sufficient for the desired length and rate of data capture. 
4. Optimize the use of the digitizer's dynamic range. PCD signals may cover several orders of magnitude, both because of a long exposure (as bubbles are eliminated) and if running experiments at varying US drive levels. It is therefore necessary to check that the signal conditioning chain scales all signals so that they can be properly recorded.

5. Include a preamplifier in the signal chain, so that the smallest expected signals can be adequately captured. In our experience, PCD self-noise is well below that of most digitizers, so a modest degree of preamplification (e.g., <100x) can still improve the SNR of the final result.

6. Filter the US source frequency prior to preamplification to avoid saturating the amplifier.

7. If an US pulser/receiver is used to provide gain and/or filtering capabilities, use it in pulser mode to confirm path length/alignment or check for unexpected scatterers in the propagation path between the PCD and the cell exposure compartment.

8. Enable real time streaming of data to storage. The SAT2 and SAT3 systems both employ 12-bit streaming USB oscilloscopes (see Table of Materials), which have the conveniences of portability and well-built user interfaces.

9. Confirm proper impedance matching in the signal chain to avoid gain or bandwidth errors. PCD devices typically have output impedances near 50 ohms, so a suitable process is to replace the PCD with a known signal from a waveform generator (with $50 \mathrm{ohm}$ output impedance) and confirm that the signal size appearing on the digitizer matches expectations, scales linearly when the injected signal is changed, and no clipping is observed for the largest signal of interest.

2. Pre-processing

1. Correct the raw voltage signals for all known gains and sensitivities in the signal path that relate to processing of data in the frequency range of interest.

2. If the data were recorded with $D C$ input coupling or otherwise show a DC-offset, remove this offset by direct subtraction or with a high pass filter.

3. Compute the power spectrum $P$ of each recorded signal.

1. Set the Fourier transform length $N_{f t}$ so that the fundamental frequency of the US source $f_{O}$ is a large integer multiple of the transform bin width: $N_{f t}$ $=n f_{S} / f_{0}$, where $n$ is an integer and $f_{S}$ is the sample frequency of the digitized data. Set $n \geq 50$ for clear capture of spectral features. For the example of a 1 $\mathrm{MHz}$ fundamental sampled at $50 \mathrm{MHz}, N_{f t}$ is 2500 and the bin width is $0.02 \mathrm{MHz}$.

2. As the transform length $N_{f t}$ is usually smaller than the duration of recorded signal, use a power spectral density (PSD) estimator such as Welch's method $^{73}$. Power in a frequency band spanning $f_{1}-f_{2}$ is $P=\int_{f_{1}}^{f_{2}} P S D d F$, where $d F$ is the transform bin width.

3. To characterize bubble activity during each exposure, estimate the contributions to the power spectrum from integer multiples of $f_{0}$ (harmonics), odd integer multiples of $f_{0} / 2$ (ultraharmonics), and broadband noise (inertial cavitation).

4. Harmonic and ultraharmonic content is most simply estimated by selecting the power spectrum values 
at specific frequencies. However, large amplitude tonal responses may spread into a small number of adjoining frequency bins (e.g. fo $\pm 2-3$ ), so these should be included in the narrow band power calculations and excluded from the broadband calculations.

5. Estimate broadband band cavitation power by subtraction of the harmonic and ultraharmonic contributions from the total power spectrum. Alternately, these contributions may be estimated using more sophisticated pre-processing ${ }^{74}$.

6. Estimate the cumulative cavitation signal energy over the duration of the exposure, preferably according to spectrum feature / bubble activity type.

7. Assuming all data records had equal duration $T_{r}$, the cumulative energy in the recorded data is $E=T_{r} \sum_{m=1}^{M} P_{m}$, where $M$ indicates the number of records.

8. If there are gaps between recordings, as may happen when the exposure is continuous, and the saved files capture a fraction of the total exposure duration $T_{e}$, the cumulative energy may be estimated as $E=\left(T_{e} / M\right) \sum_{m=1}^{M} P_{m}$

9. Estimate the measurement SNR by comparison of spectrum levels with those of background noise recorded in absence of US.

\section{Experimental Protocol}

\section{SAT Preparation}

1. Minimize the likelihood of cavitation in the propagation path by degassing the fill liquid (typically filtered water) under a pressure of $-10^{5} \mathrm{~Pa}$ for at least two hours. Confirmation with a dissolved oxygen probe that the partial pressure of oxygen is below $10 \mathrm{kPa}$ is recommended.

2. Fill the test chamber slowly to minimize reintroduction of air into the degassed liquid. Continue degassing the filled chamber if necessary.

3. Clear all residual bubbles from the transducer and media container surfaces immediately after filling and again just before initiating exposure experiments.

4. Make sure the temperature of the chamber and its contents have stabilized before commencing exposure experiments.

5. Allow the US source power amplifier to warm up (per manufacturer recommendation) so that the gain and output are stable with respect to time.

\section{Exposure Compartment Preparation}

1. Cavitation agent suspension

1. When diluting the cavitation agent, gently and continuously stir to make a uniform suspension without entrapping macrobubbles or destroying the agent (especially if they are shelled bubbles).

2. When working with MBs, withdraw and dispense slowly using the largest gauge needle available to minimize destruction during the loading process ${ }^{75}$. An $18 \mathrm{G}$ blunt fill needle has been used regularly with the SAT systems.

3. SAT2 Preparation

1. Sterilize the PDMS lid before use in experiments with live cells. 
2. Form the cell exposure compartment by press fitting the PDMS lid to the culture dish.

3. Prepare a syringe with an $18 \mathrm{G}$ blunt needle and fill with approximately $10 \mathrm{~mL}$ of liquid (e.g., MB suspension or water control).

4. Insert the needle through one of the PDMS fill holes and slowly fill the chamber, tilting so that macrobubbles can escape through the open fill hole.

For best results, tilt the chamber so that the open hole is above the fill hole.

5. When filled, close the open hole by inserting a short (4-5 $\mathrm{mm}$ ) polymer rod. Set the assembly so both holes are horizontal.

6. Remove the blunt filling needle while injecting extra fluid so that air is not drawn in. Close the hole with another polymer rod. This process completes the sealing of the cell exposure compartment.

7. Visually check the compartment for evidence of entrapped macrobubbles, and if any are found, repeat 3.3.3.-3.3.6.

8. Press fit the cell exposure compartment in the compartment holder. Install the chamber lid in place atop the chamber. Lowering the lid with an angle to horizontal discourages macrobubbles from resting on the submerged parts (absorber, holder).

9. Consider the buoyancy of the particles in suspension when deciding the orientation of the cell exposure compartment (e.g., floating bubbles or sinking nanoparticles) and how this will affect their contact with cells.
10. In all operations, use as little force as possible to minimize the flexure of the cell growth surface and detachment of cells.

\section{SAT3 Preparation}

1. Fill the Transwell with approximately $150 \mu \mathrm{L}$ of liquid (e.g., MB suspension or water control).

2. Form the cell exposure compartment by carefully sealing the transwell with a rubber plug, removing any overflow liquid with a clean paper towel or wipe. Before use in experiments with live cells, sterilize the rubber plug.

3. Visually check the compartment for evidence of entrapped macrobubbles, and if any are found, remove the plug, remove the macrobubbles and repeat 4.4.2.

4. Press fit the cell exposure compartment in the compartment holder. Install the chamber lid in place atop the chamber. Lowering the lid with an angle to horizontal discourages macrobubbles from resting on the submerged parts (absorber, holder).

NOTE: As noted above, macrobubbles may cause a variety of non-repeatable and potentially detrimental effects on US exposure experiments. Most critically, macrobubbles trapped in the cell exposure compartment may cause PCD responses and local cellular bioeffects that are not representative of the intended treatment. Always visually inspect all system components to find and remove macrobubbles before initiating US experiments.

\section{Data Collection}

1. Establish background PCD response levels by conducting initial experiments with a cell exposure 
compartment filled with control liquid (e.g., degassed water or cell media).

2. Record PCD data without driving the US source to establish background electronic noise levels.

3. Record PCD data while driving the US source at the full range of planned drive levels. This data will indicate what parts of the acoustic response are unrelated to the cavitation agents to be tested subsequently.

NOTE: Common laboratory liquids (e.g., PBS or cell media) will exhibit cavitation at moderate pressures (e.g. $0.5 \mathrm{MPa}$ at $0.5 \mathrm{MHz}$ ) if not degassed.

4. Before commencing measurements, give time for the suspension to thermally equilibrate with the chamber temperature. A fine needle thermocouple may be useful for this purpose.

5. Monitor the experiments in real time in both the time and frequency domains.

1. Time domain monitoring of the PCD reveals whether signals are sized appropriately for the current instrumentation settings. Specifically, signal clipping is to be avoided, since it will appear in the frequency domain as multi-tone harmonic response.

2. Time domain monitoring of the PCD also shows if cavitation signals are seen earlier than expected based on propagation time from the US source to the exposure compartment to the PCD. If such signals are seen, this may indicate leakage of cavitation agent into the test chamber.

3. Frequency domain monitoring of the PCD indicates the type of bubble behavior and can be used to adjust the drive levels as needed to achieve the desired cell stimulus (e.g., lower drive levels for harmonic excitation).
6. To ensure that the first exposures are not missed, start the data collection process prior to turning on the US source drive signal.

7. Monitor the amplifier output signal that drives the US source (as opposed to the waveform generator output) throughout the experiment to ensure that the exposure is proceeding as expected. Use a high voltage probe for this measurement and make sure that the oscilloscope is set to compensate for probe attenuation.

8. After exposing a sample, carefully remove it from the test chamber.

1. Remove and clean the PDMS lid (SAT2)/rubber plug (SAT3) in preparation for any subsequent use.

2. Transfer the culture dish (SAT2)/Transwell (SAT3) as needed for subsequent analysis (e.g., microscopy, fluorescence imaging).

3. After a small number of exposures (e.g., 3-5), it is good practice to re-acquire baseline cavitation signals (in the absence of cavitation agent) and compare with the original data set to make sure the chamber media have not been contaminated.

\section{Representative Results}

Figure 4 shows examples of time and frequency domain PCD responses, illustrating three distinct cavitation behaviors. All data were collected on SAT3 using SonoVue MBs diluted $5 x$ in PBS, with a final concentration of $\sim 2^{*} 10^{7} \mathrm{MBs} / \mathrm{ml}$. The temperature for all examples in this section was $19 \pm 1{ }^{\circ} \mathrm{C}$. The US source was driven with a $2.0 \mathrm{~ms}$ pulse at $0.5 \mathrm{MHz}$ to achieve incident peak negative pressures of 0.20 (Figure 4A and 4B), 0.30 (Figure 4C and 4D), and $0.70 \mathrm{MPa}$ (Figure $4 \mathrm{E}$ and $4 \mathrm{~F}$ ). The signal recordings began $1.4 \mathrm{~ms}$ before the $t=0$ start of the US pulse. The inset traces show the signal 
as recorded (red) and with a $2 \mathrm{MHz}$ high pass filter (blue) for a time window centered at the time of flight from source to cell exposure compartment to PCD. The low-level response before this time is due to directly received radiation from the source, which is common in configurations where the PCD is behind the US source.

At the lowest incident pressure, the PCD response consists entirely of integer harmonics of the $0.5 \mathrm{MHz}$ fundamental US frequency. Increasing from 0.20 to $0.30 \mathrm{MPa}$ results in pronounced ultraharmonics in the spectrum in addition to further elevated integer harmonics. The time domain waveforms at these two pressures look similar, although the $0.30 \mathrm{MPa}$ results show more variability over the pulse duration. At the highest pressure, the time domain waveform amplitude has grown nonlinearly relative to the lower pressures as a result of clearly elevated broadband noise visible in the spectrum. This noise is commonly considered to be a result of inertial cavitation and in this example, corresponds to destruction of MBs.

To see this more clearly, PCD responses as a function of time are shown in Figure 5. In the left panel (Figure 5A), full spectra are shown over a 50 second exposure time, during which the source emitted 2.0 ms pulses every 0.20 seconds. Corresponding total, harmonic and broadband powers are shown in the right panel (Figure 5B). The US was turned on at $\mathrm{t}=3.0 \mathrm{~s}$, at which time large-amplitude broadband responses were seen. The initial spike is thought to correspond to the destruction of the largest bubbles in the suspension (SonoVue is polydisperse) and is a common observation in cavitation experiments with shelled bubbles and even with non-degassed media (e.g., PBS).

After a few seconds, the broadband response rapidly diminished, apparently due to bubble destruction, and the signal is predominantly comprised of harmonics. This suggests that the freed gas and remaining MBs are vibrating stably and non-inertially. At $\mathrm{t}$ 50s, the broadband component has fallen to the level of the original background noise. Exposure tests like this are therefore important when trying to understand the timescales during which different bubble effects may be acting upon the cells in the chamber.

Bubbles are likely to translate in response to radiation forces generated during US exposure and movement of MBs in and out of the PCD field of view can lead to increased variability in the monitored cavitation signal, especially when dealing with dilute suspensions. The sensitive region of the PCD should therefore span as much of the cell exposure surface as possible. A comparison of the responses focused and unfocused PCDs with identical center frequencies (see Figure 2) is shown in Figure 6, using a 20:1 dilution of MBs in normal PBS is SAT2. The time and sample-averaged spectra in panel Figure 6A show that the unfocused PCD contains a stronger broadband response, accompanied by reduced sample-to-sample variability in both harmonic (Figure 6B) and ultraharmonic powers (Figure 6C).

It is important to recognize that media used for in vitro cell work are not degassed and may present an enhanced background level of bubble activity. Figure 7 shows the response in SAT2 of PBS used in its supplier-provided form and after two hours of degassing under vacuum, after which the air saturation was reduced from $92 \%$ to $46 \%$ as determined with an optical sensor (PreSens, Germany). The spectra in Figure 7A were averaged over exposure time and repeats with five independent samples, and clearly show show clearly elevated ultraharmonics in normal PBS. Powers summed over three harmonics (Figure 7B) are well within the standard deviation of each experimental output. 
By contrast, the ultraharmonic sums in Figure 7C show that normal PBS has nearly an order of magnitude higher level and substantially higher variability between samples. These examples indicate that a common cell-compatible medium may exhibit behaviors that could be (incorrectly) attributed to the presence of MBs. Since it is usually impractical to degas culture medium due to the negative impact upon cells and/ or cavitation agent stability, it is critical to perform suitable controls in any cavitation-related study.

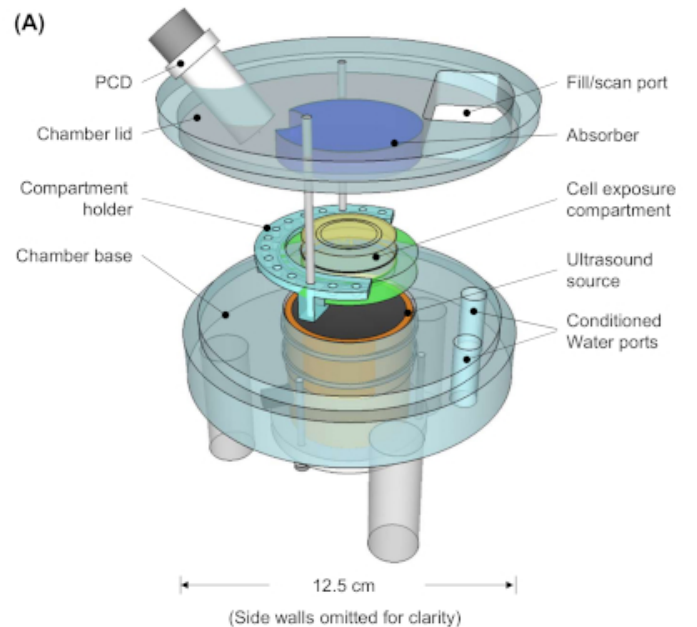

(B)

(D)

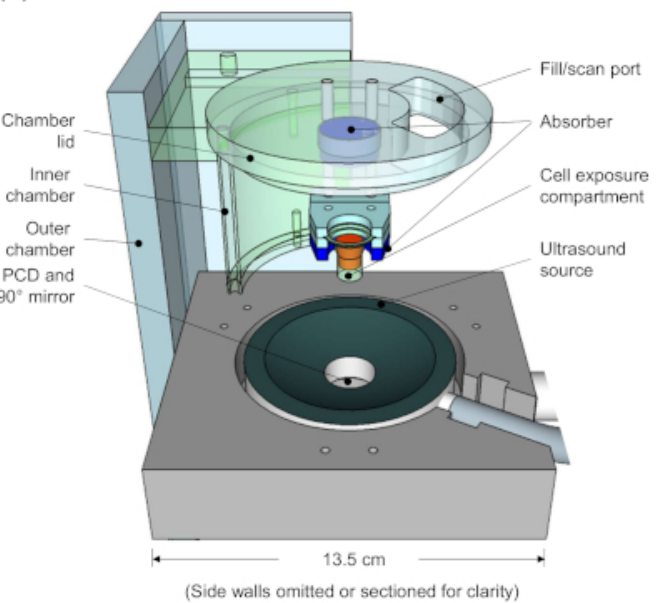

(E)
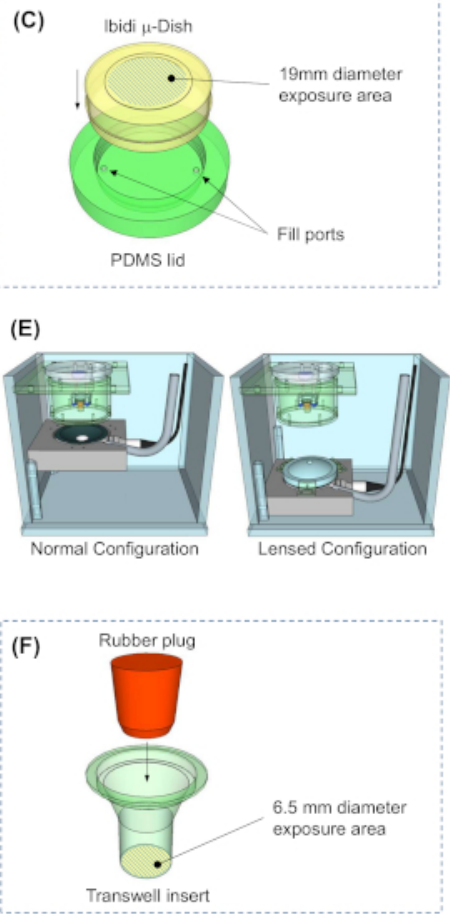

Figure 1: Illustrations of two US exposure system designs incorporating cavitation monitoring: SAT3 (D-F). (A) SAT2 annotated assembly with side wall removed for clarity. (B) SAT2 with side wall intact. (C) SAT2 cell exposure compartment, disassembled. (D) SAT3 annotated assembly. (E) SAT3 in normal (left) and lensed (right) configurations for beam width matching at different frequencies. (F) SAT3 cell exposure compartment, disassembled. Please click here to view a larger version of this figure. 

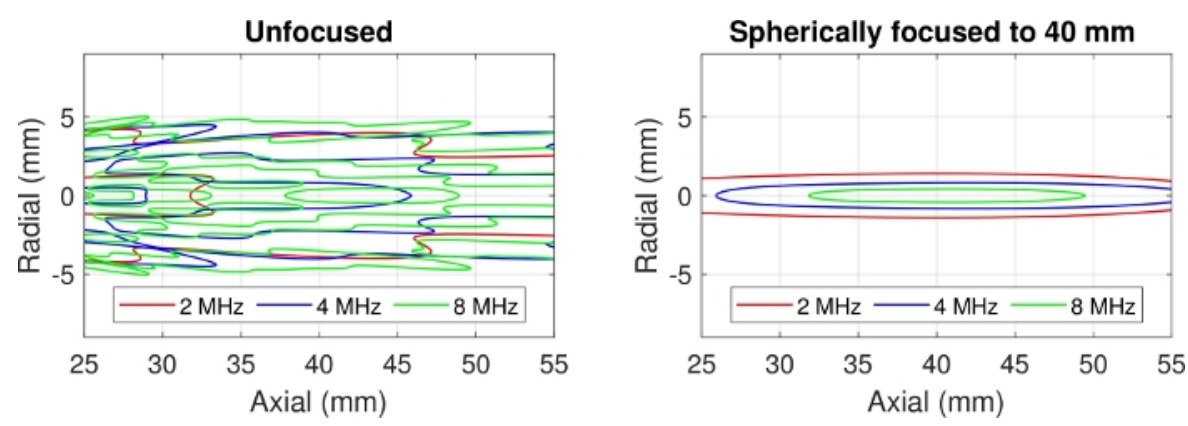

Figure 2: Calculations of half amplitude pressure field contours for $12.7 \mathrm{~mm}$ diameter unfocused (left) and spherically focused (right) transducers. Frequencies of 2, 4 and $8 \mathrm{MHz}$ are shown as red, blue, and green contours, respectively, for a PCD element at the coordinate origin $(0,0)$. The outermost contours of the unfocused device are relatively insensitive to frequency, but the interior structure is frequency dependent. The spherically focused field contracts as frequency increases, but inside the contours, the fields vary smoothly. Please click here to view a larger version of this figure.

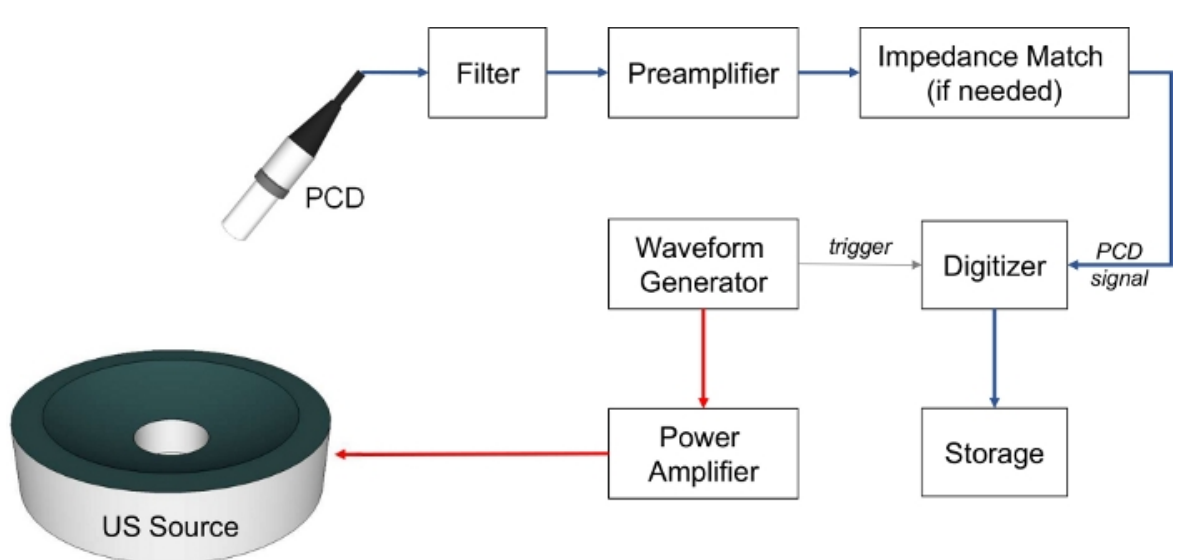

Figure 3: Instrumentation for cavitation signal conditioning and recording (blue arrows), US source excitation (red lines), and data acquisition triggering. Please click here to view a larger version of this figure. 


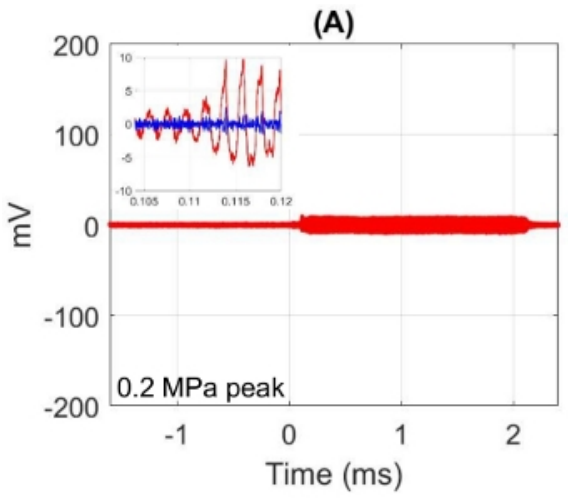

(C)

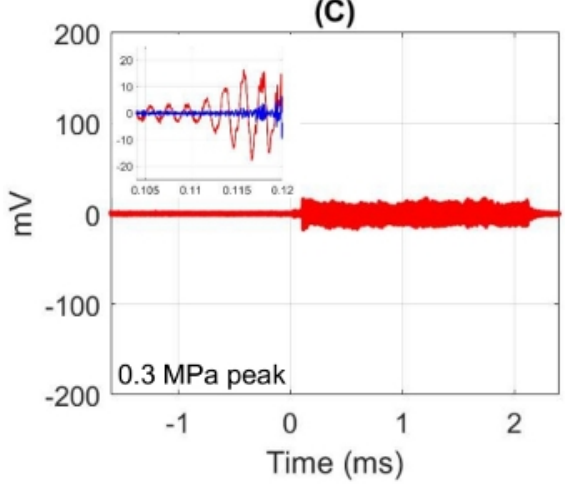

(E)

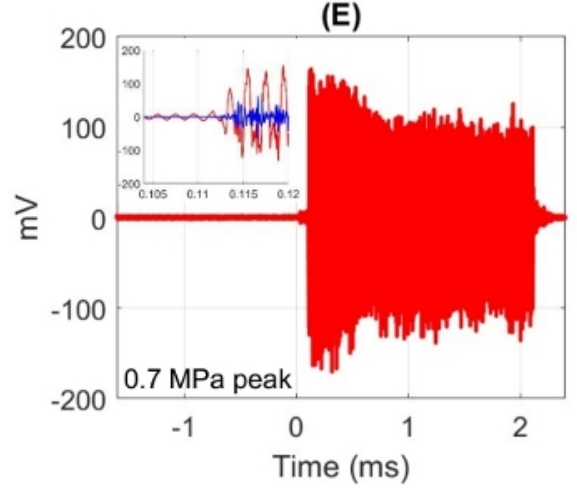

(B)

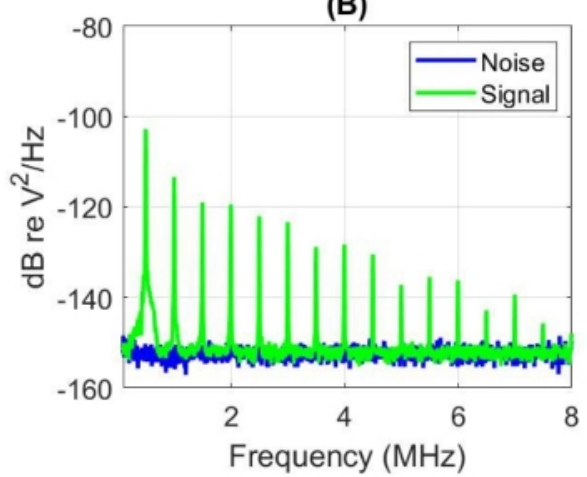

(D)

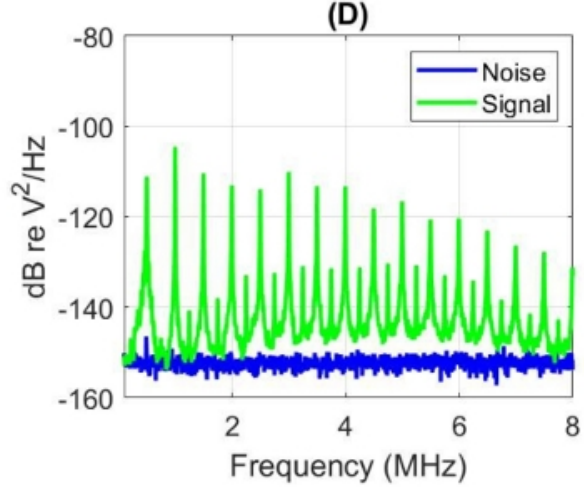

(F)

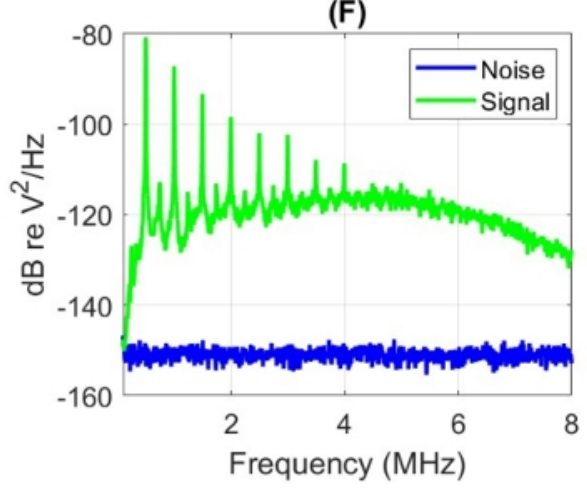

Figure 4: Time (left) and frequency (right) domain PCD responses recorded with MBs diluted 5x in PBS. Incident peak negative pressures were (A, B) 0.2 MPa, (C, D) $0.4 \mathrm{MPa},(E, F) 0.7 \mathrm{MPa}$, all at $0.5 \mathrm{MHz}$. Signal recordings begin $1.4 \mathrm{~ms}$ before the $t=0$ start of the 2.0 ms duration ultrasound pulse. (A, C, E) Time domain signals (red) are shown on a fixed vertical scale, indicating how the response level changes with incident pressure. The inset traces show the signal as recorded (red) and with a $2 \mathrm{MHz}$ high pass filter (blue) for a time window centered at the time of flight from source to cell exposure compartment to PCD. (B, D, F) Noise and signal power spectral densities are calculated for $t<0$ and $t>0$, respectively. Please click here to view a larger version of this figure. 

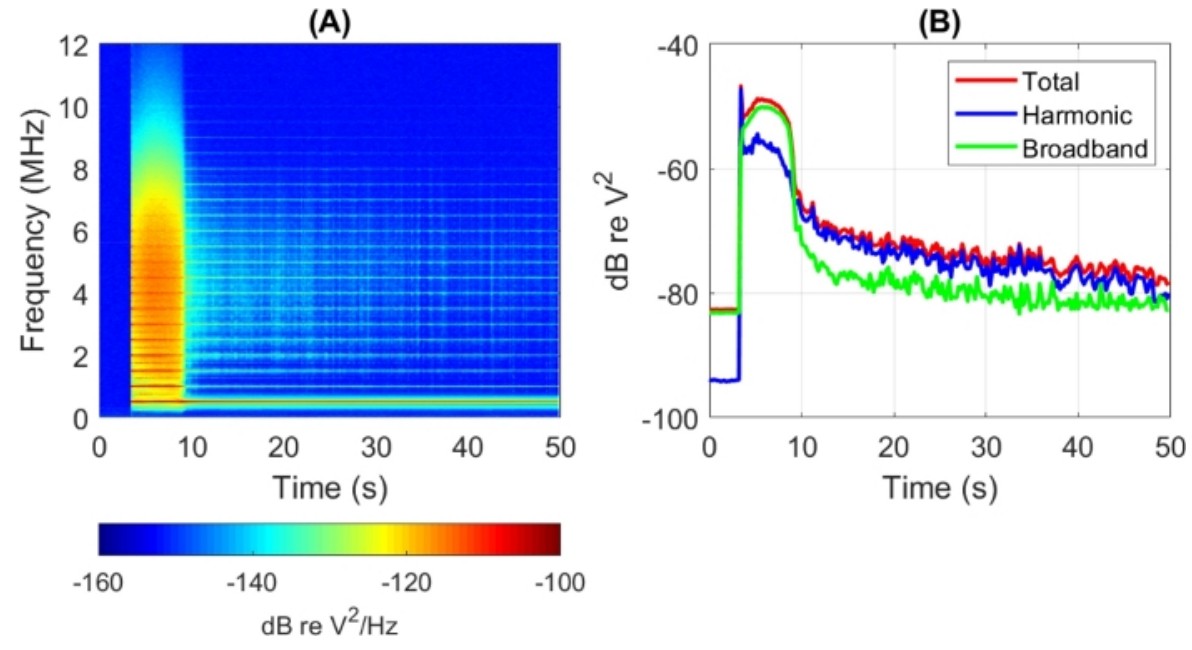

Figure 5: Spectrum histories over a 50 second exposure of a suspension of MBs diluted 5x in PBS. (A) Full spectra and (B) total, harmonic and broadband signal powers, all as a function of time. Drive conditions were $0.5 \mathrm{MHz}, 0.7 \mathrm{MPa}$ peak negative pressure, $2.0 \mathrm{~ms}$ pulse duration, $200 \mathrm{~ms}$ pulse repetition period. Please click here to view a larger version of this figure.
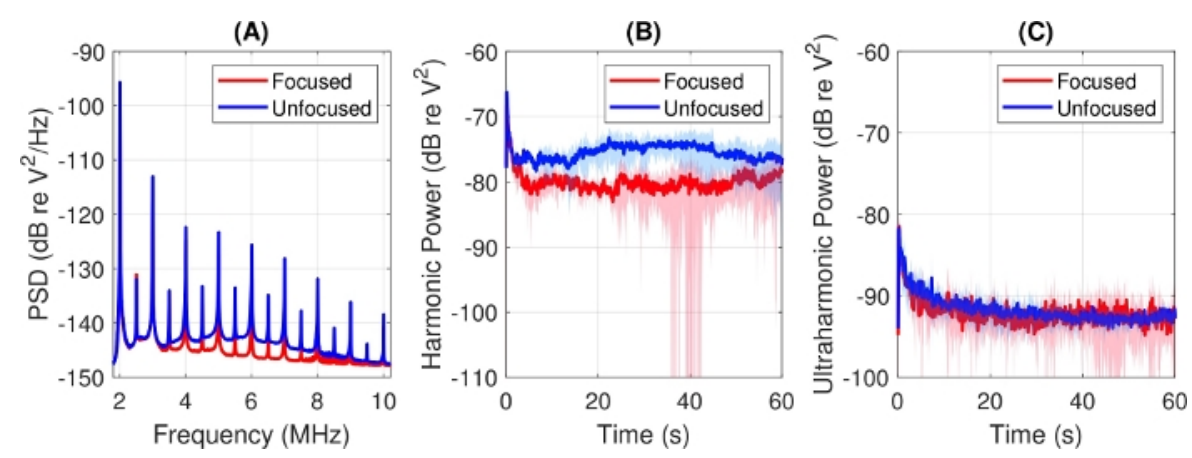

Figure 6: Effect of PCD focusing geometry recorded with a 20:1 dilution of microbubbles in normal PBS. Drive conditions were: $1.0 \mathrm{MHz}, 0.50 \mathrm{MPa}$ peak negative pressure, $3.0 \mathrm{~ms}$ pulse duration, $10 \mathrm{~ms}$ pulse repetition period. (A) Full spectra averaged over exposure time and three independent sample repeats. (B) Power in 3, 4 and $5 \mathrm{MHz}$ harmonics, and (C) power in 2.5, 3.5 and 4.5 MHz ultraharmonics. Thick lines are sample means, shaded areas indicate $+/-1$ standard deviation. Please click here to view a larger version of this figure. 

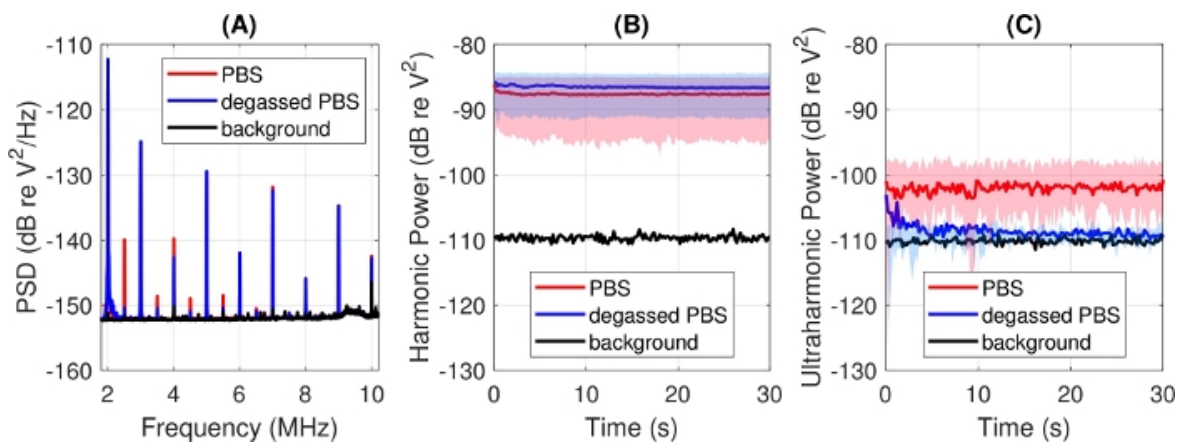

Figure 7: Effect of degassed media recorded with PBS. (A) Full spectra averaged over exposure time and five independent sample repeats. (B) Power in 3, 4 and $5 \mathrm{MHz}$ harmonics, and (C) power in 2.5, 3.5 and $4.5 \mathrm{MHz}$ ultraharmonics. Thick lines are sample means, shaded areas indicate +/- 1 standard deviation. Drive conditions were $1.0 \mathrm{MHz}, 0.50 \mathrm{MPa}$ peak negative pressure, $1.0 \mathrm{~ms}$ pulse duration, $200 \mathrm{~ms}$ pulse repetition period. Please click here to view a larger version of this figure.

\begin{tabular}{|c|c|c|c|}
\hline Parameter & Unit & Minimum & Maximum \\
\hline frequency & $\mathrm{MHz}$ & 0.02 & 20 \\
\hline pressure (peak negative) & $\mathrm{MPa}$ & 0.1 & $\mathrm{CW}$ \\
\hline pulse length & cycles & 1 & $\mathrm{CW}$ \\
\hline duty cycle & $\%$ & 10 & 1000 \\
\hline
\end{tabular}

Table 1: Summary of the range of reported parameters facilitating sonoporation in vitro.

\section{Discussion}

The critical steps for any acoustic measurement were encapsulated by Apfel in $1981^{76}$ as "know thy liquid, know thy sound field, know when something happens." In the context of this protocol, these encompass the transducer calibration and alignment and the water preparation and bubble handling steps. First, it is essential that the hydrophone used to calibrate the driving transducer and/or the PCD is itself accurately calibrated through regular external servicing or in-house comparison to a reference standard. Similarly, the response of both the driving transducer and PCD need to be regularly characterized to check for any change in output and/or loss of sensitivity. If the driving conditions and receive sensitivity of the system are unknown, then it will be impossible to infer any meaningful relationship between exposure conditions, bioeffects and acoustic emissions. Directly related to this, the alignment of the transducers to each other and the sample chamber needs to be carefully 
checked to ensure that the exposure conditions within the chamber are as expected and the sampling volume for the PCD corresponds to the region of interest. As indicated, the temperature and gas content of the suspending medium can affect the final results significantly and consistency is extremely important in this respect ${ }^{77,78}$. Similarly, the preparation, characterization, and handling of the cavitation agent suspension require very close attention to ensure that the expected size distribution and concentration of particles is present within the sample. For example, if the concentration of bubbles is too high, there will be effective shielding of the sample volume from the incident US field. MB agents are particularly susceptible to destruction and coalescence and further guidance on their handling may be found in Mulvana et. al. $(2012)^{79}$.

A very common problem with detection of cavitation signals is achieving an adequate SNR. This is due partly to the nature of the signal itself, as described, but may also be due to sources of electrical noise within the experimental set up. Checking the connections between system components, in particular those involving co-axial cables, may help to eliminate some of these. Replacing or repairing co-axial cables may be necessary. Identifying and removing or deactivating other equipment in the laboratory such as pumps that may cause electrical noise can also help. Poor electrical impedance matching between system components can be a further cause of poor signal to noise ratio and also potentially of damage to equipment and should be carefully checked. The triggering settings on the signal generator and oscilloscope should similarly be checked to confirm that they are configured appropriately for the experiment and have not reverted to the manufacturer default settings. If there is significant destruction of bubbles during handling, in case of the SAT2, it may be helpful to attach a second syringe to the outlet port and use this to gently extract fluid from the chamber, thereby drawing in the suspension. This can also help in eliminating macrobubbles or enabling flow during US exposure, if desired.

It is not possible to completely eliminate acoustic reflections within the sample chamber and hence the incident field will not be completely uniform over the whole sample volume. As mentioned in steps 1.3.2 and 1.3.3, the transmissibility of acoustic windows will be frequency dependent and thus the desired bandwidth for acoustic emission measurements should be carefully considered. In particular, there may be significant multiple reflections of higher frequency components. This is another reason why calibration of the field within the fully assembled system is so important for minimizing the uncertainty in incident pressure. Appropriate gating of the recorded signals should also be considered to minimize the effects of multiple reflections. The use of commercial devices for convenience and the need for acoustic transparency means that some optical transparency must be sacrificed. This may impact the quality of subsequent imaging, e.g., to assess cell viability or drug uptake. Some of the membranes used in commercial devices are also porous and, thus, imperfect isolation occurs between the sample chamber and the surrounding water bath. As above, the corresponding risk of contamination can be mitigated by using a smaller sub-chamber, the contents of which can be regularly replaced. The cell culture devices indicated in the Table of Materials are suitable primarily for cell monolayers that may not be representative of tissues in terms of all US/cavitationmediated bioeffects. The proximity of the cells to a solid surface will also affect MB dynamics in a way that may not be reflective of conditions in vivo, e.g., promoting microstreaming and microjetting as described in the introduction. These 
limitations can be addressed, however, through a simple substitution of alternative tissue models.

The aim in proposing the SATs is to provide a means of improving the reproducibility of acoustic exposure conditions and acoustic emissions between studies of US-mediated bioeffects, thus hopefully facilitating better understanding of the underlying mechanisms and the development of treatment monitoring techniques to improve safety and efficacy. The systems are designed to be compatible with commercially available cell culture devices, enabling a wide range of biological assays to be performed according to the application of interest and enabling the performance of high throughput experiments, removing the need for time consuming alignment procedures between runs. By standardizing protocols for the characterization of exposure conditions and the capture of acoustic emissions, the system dependent variability can hopefully be reduced. The range of parameters that should be explored for a particular experiment will depend upon the application (desired bioeffect, cell type, depth of target tissue if in vivo etc.) and the nature of any cavitation agent being used. Given the large number of variables (US frequency, pressure amplitude, pulse length, pulse repetition frequency etc.) fully exploring the whole parameter space is unlikely to be practicable. An advantage of the proposed protocol is that it enables some bounds on this parameter space to be quickly established. For example, it enables determination of the minimum pressure at which a cavitation signal is generated, the maximum pressure or pulse length that can be used before cell detachment/death occurs, and the pressure at which fractional harmonics or broadband noise are produced. It is recommended that such a set of scoping measurements be carried out as a first step in any study.
As presented, the SATs are designed for real-time monitoring of acoustic emissions, with biological assays being performed outside of the experiment. It would be relatively simple, however, to modify the SAT to enable direct optical observation of the sample chamber via a microscope objective. This could in turn be coupled to a fluorescence and/ or high-speed microscopy system to enable observation of drug uptake and bubble dynamics, for example. The PCD output as currently presented in terms of voltage indicates: i) the types of cavitation behavior and their relative proportions; ii) how long these cavitation behaviors persist; iii) whether the observed time-cumulative exposure characteristics are correlated to a particular bioeffect; and iv) whether the relative levels and time-dependent behaviors are consistent with previous experiments in the exposure system. Whilst the receive sensitivity of the $\mathrm{PCD}$ can be quantified, in order to reliably characterize the acoustic emissions in terms of absolute energy, additional spatial information is required. This could be achieved by replacing the PCD with an array probe to implement passive acoustic mapping (PAM) ${ }^{80}$. This would, however increase the complexity of signal processing and the computational time and power required.

Other instrumentation for measurement of membrane electrical resistance or application of physical targeting methods, for example magnetic fields, could also be incorporated. It would also be possible to use threedimensional tissue structures such as tumor spheroids, organoids, or even ex vivo tissue samples on acoustically "soft" gel substrates in place of the cell monolayers to study US and cavitation-mediated effects in more realistic tissue environments. 


\section{Disclosures}

The authors have nothing to disclose.

\section{Acknowledgments}

The authors thank the Engineering and Physical Sciences Research Council for supporting this work through grant EP/L024012/1. VB is also supported by the Engineering and Physical Sciences Research Council (EPSRC) and Medical Research Council (MRC) (grant EP/L016052/1). VB and AV thank the Clarendon Foundation for Post Graduate Scholarships. AV also thanks Exeter College for a Santander scholarship. The authors are indebted to James Fisk and David Salisbury for their invaluable assistance in the manufacturing of the apparatus. They also gratefully acknowledge the contributions of Drs. Dario Carugo and Joshua Owen in the development of earlier prototype SATs.

\section{References}

1. Maier, A., Steidl, S., Christlein, V., Hornegger, J. Medical Imaging Systems - An Introductory Guide. Lecture Notes in Computer Science. Springer. (2018).

2. Tachibana, K., Tachibana, S. Albumin microbubble echo-contrast material as an enhancer for ultrasound accelerated thrombolysis. Circulation. 92, 1148-1150 (1995).

3. Bao, S., Thrall, B.D., Miller, D.L. Transfection of a reporter plasmid into cultured cells by sonoporation in vitro. Ultrasound in Medicine and Biology. 23 (6), 953-9 (1997).

4. Price, R.J., Skyba, D.M., Kaul, S., Skalak, T.C. Delivery of colloidal particles and red blood cells to tissue through microvessel ruptures created by targeted microbubble destruction with ultrasound. Circulation. 98 (13), 1264-1267 (1998).

5. Theek, B. et al. Sonoporation enhances liposome accumulation and penetration in tumors with low EPR. Journal of Controlled Release. 231, 77-85 (2016).

6. Dimcevski, G. et al. A human clinical trial using ultrasound and microbubbles to enhance gemcitabine treatment of inoperable pancreatic cancer. Journal of Controlled Release. 243, 172-181 (2016).

7. Snipstad, S. et al. Ultrasound Improves the Delivery and Therapeutic Effect of Nanoparticle-Stabilized Microbubbles in Breast Cancer Xenografts. Ultrasound in Medicine and Biology. 43 (11), 2651-2669 (2017).

8. Unga, J., Hashida, M. Ultrasound induced cancer immunotherapy. Advanced Drug Delivery Reviews. 72, 144-53 (2014)

9. Yang, C., Du, M., Yan, F., Chen, Z. Focused ultrasound improves NK-92Ml cells infiltration into tumors. Frontiers in Pharmacology. 10, 326 (2019).

10. McDannold, N., Arvanitis, C.D., Vykhodtseva, N., Livingstone, M.S. Temporary disruption of the bloodbrain barrier by use of ultrasound and microbubbles: Safety and efficacy evaluation in rhesus macaques. Cancer Research. 72 (14), 3652-3663 (2012).

11. O'Reilly, M.A., Hynynen, K. Ultrasound and microbubblemediated blood-brain barrier disruption for targeted delivery of therapeutics to the brain. Methods in Molecular Biology. 1831, 111-119 (2018).

12. Mainprize, T. et al. Blood-Brain Barrier Opening in Primary Brain Tumors with Non-invasive MR-Guided Focused Ultrasound: A Clinical Safety and Feasibility Study. Scientific Reports. 9, 321 (2019). 
13. Ebben, H.P., Nederhoed, J.H., Lely, R.J., Wisselink, W., Yeung, K. Microbubbles and UltraSound-accelerated Thrombolysis (MUST) for peripheral arterial occlusions: Protocol for a phase II single-arm trial. BMJ Open. 7, e014365 (2017).

14. de Saint Victor, M., Barnsley, L.C., Carugo, D., Owen, J., Coussios, C.C., Stride, E. Sonothrombolysis with Magnetically Targeted Microbubbles. Ultrasound in Medicine \& Biology. 45 (5), 1151-1163 (2019).

15. Dixon, A.J., Li, J., Rickel, J.M.R., Klibanov, A.L., Zuo, Z., Hossack, J.A. Efficacy of Sonothrombolysis Using Microbubbles Produced by a Catheter-Based Microfluidic Device in a Rat Model of Ischemic Stroke. Annals of Biomedical Engineering. (2019).

16. Horsley, H. et al. Ultrasound-activated microbubbles as a novel intracellular drug delivery system for urinary tract infection. Journal of Controlled Release. 301, 166-175 (2019).

17. Lattwein, K.R., et al. Sonobactericide: An Emerging Treatment Strategy for Bacterial Infections. Ultrasound in Medicine and Biology. 46 (2), 193-215 (2020).

18. Crum, L.A., Fowlkes, J.B. Acoustic cavitation generated by microsecond pulses of ultrasound. Nature. 319, 52-54 (1986).

19. Holland, C.K., Apfel, R.E. Thresholds for transient cavitation produced by pulsed ultrasound in a controlled nuclei environment. Journal of the Acoustical Society of America. 88, 2059-69 (1990).

20. Rifai, B., Arvanitis, C.D., Bazan-Peregrino, M., Coussios, C.C. Cavitation-enhanced delivery of macromolecules into an obstructed vessel. The Journal of the Acoustical Society of America. 128, EL310 (2010).
21. Wu, J., Ross, J.P., Chiu, J.F. Reparable sonoporation generated by microstreaming. The Journal of the Acoustical Society of America. 111 (3), 1460-1464 (2002).

22. Doinikov, A.A., Bouakaz, A. Acoustic microstreaming around a gas bubble. The Journal of the Acoustical Society of America. 127 (2), 703-709 (2010).

23. De Cock, I. et al. Ultrasound and microbubble mediated drug delivery: acoustic pressure as determinant for uptake via membrane pores or endocytosis. Journal of Controlled Release: Official Journal of the Controlled Release Society. 197, 20-28 (2015).

24. Pereno, V., Lei, J., Carugo, D., Stride, E. Microstreaming inside Model Cells Induced by Ultrasound and Microbubbles. Langmuir. 36, 6388-6398 (2020).

25. Chen, H., Brayman, A.A., Kreider, W., Bailey, M.R., Matula, T.J. Observations of translation and jetting of ultrasound-activated microbubbles in mesenteric microvessels. Ultrasound in Medicine and Biology. 37 (12), 2139-48 (2011).

26. Lentacker, I., De Smedt, S.C., Sanders, N.N. Drug loaded microbubble design for ultrasound triggered delivery. Soft Matter. 5, 2161-2170 (2009).

27. Song, J.H., Moldovan, A., Prentice, P. Non-linear Acoustic Emissions from Therapeutically Driven Contrast Agent Microbubbles. Ultrasound in Medicine and Biology. 45 (8), 2188-2204 (2019).

28. Ohl, C., Arora, M., Ikink, R., Jong, N. De, Versluis, M., Delius, M. Sonoporation from Jetting Cavitation Bubbles. Biophysical Journal. 91 (11), 4285-4295 (2006).

29. Li, Z.G., Liu, a Q., Klaseboer, E., Zhang, J.B., Ohl, C.D. Single cell membrane poration by bubble-induced 
microjets in a microfluidic chip. Lab on a Chip. 13 (6), 1144-1150 (2013).

30. Wang, Q.X., Manmi, K. Three dimensional microbubble dynamics near a wall subject to high intensity ultrasound. Physics of Fluids. 26, 032104 (2014).

31. Suslick, K.S. Ultrasound: Its Chemical, Physical, and Biological Effects. Radiology. VHC Publishers. New York. (1988).

32. Mitragotri, S. Healing sound: the use of ultrasound in drug delivery and other therapeutic applications. Nature reviews. Drug discovery. 4 (3), 255-260 (2005).

33. Mitragotri, S. Sonophoresis: Ultrasound-mediated transdermal drug delivery. Percutaneous Penetration Enhancers Physical Methods in Penetration Enhancement. 3-14 (2017).

34. Park, J., Lee, et al. Enhanced Transdermal Drug Delivery by Sonophoresis and Simultaneous Application of Sonophoresis and Iontophoresis. AAPS PharmSciTech. 20 (3), 96 (2019).

35. Lentacker, I., De Cock, I., Deckers, R., De Smedt, S.C., Moonen, C.T.W. Understanding ultrasound induced sonoporation: Definitions and underlying mechanisms. Advanced Drug Delivery Reviews. 72, 49-64 (2014).

36. Wawryka, P., Kiełbik, A., Iwanek, G. Microbubble based sonoporation - from the basics into clinical implications. Medical Research Journal. 4 (3), 178-183 (2019).

37. Hilgenfeldt, S., Lohse, D., Zomack, M. Sound scattering and localized heat deposition of pulse-driven microbubbles. The Journal of the Acoustical Society of America. 107 (6), 3530-3539 (2000).

38. Holt, R.G., Roy, R.A. Measurements of bubble-enhanced heating from focused, MHz-frequency ultrasound in a tissue-mimicking material. Ultrasound Medical Biology. 27 (10), 1399-1412 (2001).

39. Tan, J., Li, P., Xue, H., Li, Q. Cyanidin-3-glucoside prevents hydrogen peroxide ( $\mathrm{H} 2 \mathrm{O} 2$ )-induced oxidative damage in HepG2 cells. Biotechnology Letters. 42 (11), 2453-2466 (2020).

40. Costley, D. et al. Treating cancer with sonodynamic therapy: A review. International Journal of Hyperthermia. 31 (2), 107-117 (2015).

41. You, D.G. et al. ROS-generating TiO2 nanoparticles for non-invasive sonodynamic therapy of cancer. Scientific Reports. 6, 23200 (2016).

42. Canavese, G. et al. Nanoparticle-assisted ultrasound: A special focus on sonodynamic therapy against cancer. Chemical Engineering Journal. 340, 155-172 (2018).

43. Beguin, E., et al. Direct Evidence of Multibubble Sonoluminescence Using Therapeutic Ultrasound and Microbubbles. ACS Applied Materials \& Interfaces. 11 (22), 19913-19919 (2019).

44. Stride, E. et al. Microbubble Agents: New Directions. Ultrasound in Medicine and Biology. 46 (6), 1326-1343 (2020).

45. Rapoport, N., Gao, Z., Kennedy, A. Multifunctional nanoparticles for combining ultrasonic tumor imaging and targeted chemotherapy. Journal of the National Cancer Institute. 99 (14), 1095-1106 (2007).

46. Cao, Y. et al. Drug release from phase-changeable nanodroplets triggered by low-intensity focused ultrasound. Theranostics. 8 (5), 1327-1339 (2018).

47. Zhang, L. et al. Mitochondria-Targeted and UltrasoundActivated Nanodroplets for Enhanced Deep-Penetration 
Sonodynamic Cancer Therapy. ACS Applied Materials \& Interfaces. 11 (9), 9355-9366 (2019).

48. Delogu, L.G. et al. Functionalized multiwalled carbon nanotubes as ultrasound contrast agents. Proceedings of the National Academy of Sciences of the United States of America. 109 (41), 16612-16617 (2012).

49. Paris, J.L. et al. Ultrasound-mediated cavitationenhanced extravasation of mesoporous silica nanoparticles for controlled-release drug delivery. Chemical Engineering Journal. 340, 2-8 (2018).

50. Mannaris, C., et al. Gas-Stabilizing Gold Nanocones for Acoustically Mediated Drug Delivery. Advanced Healthcare Materials. 7 (12), 1800184 (2018).

51. Kwan, J.J., et al. Ultrasound-induced inertial cavitation from gas-stabilizing nanoparticles. Physical Review E Statistical, Nonlinear, and Soft Matter Physics. 92 (2) (2015).

52. Kwan, J.J. et al. Ultrasound-Propelled Nanocups for Drug Delivery. Small. 11 (39), 5305-5314 (2015).

53. Mannaris, C. et al. Microbubbles, Nanodroplets and Gas-Stabilizing Solid Particles for Ultrasound-Mediated Extravasation of Unencapsulated Drugs: An Exposure Parameter Optimization Study. Ultrasound in Medicine and Biology. 45, 954-967 (2019).

54. Roovers, S. et al. The Role of Ultrasound-Driven Microbubble Dynamics in Drug Delivery: From Microbubble Fundamentals to Clinical Translation. Langmuir. 35, 10173-10191 (2019).

55. Lentacker, I., Geers, B., Demeester, J., De Smedt, S.C., Sanders, N.N. Design and Evaluation of Doxorubicin-containing Microbubbles for Ultrasoundtriggered Doxorubicin Delivery: Cytotoxicity and
Mechanisms Involved. Molecular Therapy. 18 (1), 101-108 (2010).

56. De Cock, I., Lajoinie, G., Versluis, M., De Smedt, S.C., Lentacker, I. Sonoprinting and the importance of microbubble loading for the ultrasound mediated cellular delivery of nanoparticles. Biomaterials. 83, 294-307 (2016).

57. Roovers, S. et al. Sonoprinting of nanoparticleloaded microbubbles: Unraveling the multi-timescale mechanism. Biomaterials. 217, 119250 (2019).

58. Carugo, D. et al. Modulation of the molecular arrangement in artificial and biological membranes by phospholipid-shelled microbubbles. Biomaterials. 113, 105-117 (2017).

59. Stride, E.P., Coussios, C.C. Cavitation and contrast: The use of bubbles in ultrasound imaging and therapy. Proceedings of the Institution of Mechanical Engineers, Part H: Journal of Engineering in Medicine. 224 (2), 171-91 (2010).

60. Stride, E., Coussios, C. Nucleation, mapping and control of cavitation for drug delivery. Nature Reviews Physics. 1, 495-509 (2019).

61. Dong, Y., et al. Antibiofilm effect of ultrasound combined with microbubbles against Staphylococcus epidermidis biofilm. International Journal of Medical Microbiology. 307 (6), 321-328 (2017).

62. Van Rooij, T., et al. Vibrational Responses of Bound and Nonbound Targeted Lipid-Coated Single Microbubbles. IEEE Transactions on Ultrasonics, Ferroelectrics, and Frequency Control. 64 (5), 785-797 (2017).

63. Duan, X., Yu, A.C.H., Wan, J.M.F. Cellular Bioeffect Investigations on Low-Intensity Pulsed Ultrasound 
and Sonoporation: Platform Design and Flow Cytometry Protocol. IEEE Transactions on Ultrasonics, Ferroelectrics, and Frequency Control. 66 (9), 1422-1434 (2019).

64. Hu, Y., Wan, J.M.F., Yu, A.C.H. Membrane Perforation and Recovery Dynamics in Microbubble-Mediated Sonoporation. Ultrasound in Medicine and Biology. 39 (12), 2393-2405 (2013).

65. Carugo, D., Owen, J., Crake, C., Lee, J.Y., Stride, E. Biologicallyand acoustically compatible chamber for studying ultrasound-mediated delivery of therapeutic compounds. Ultrasound in Medicine and Biology. 41 (7), 1927-1937 (2015).

66. Pereno, V. et al. Layered acoustofluidic resonators for the simultaneous optical and acoustic characterisation of cavitation dynamics, microstreaming, and biological effects. Biomicrofluidics. 12 (3), 034109 (2018).

67. Fan, Z., Liu, H., Mayer, M., Deng, C.X.C.X. Spatiotemporally controlled single cell sonoporation. Proceedings of the National Academy of Sciences of the United States of America. 109 (41), 16486-16491 (2012).

68. Helfield, B., Chen, X., Watkins, S.C., Villanueva, F.S. Biophysical insight into mechanisms of sonoporation. Proceedings of the National Academy of Sciences. (2016).

69. Helfield, B.L., Chen, X., Qin, B., Watkins, S.C., Villanueva, F.S. Mechanistic Insight into Sonoporation with Ultrasound-Stimulated Polymer Microbubbles. Ultrasound in Medicine and Biology. 43 (11), 2678-2689 (2017).

70. Aron, M., Vince, O., Gray, M., Mannaris, C., Stride, E. Investigating the Role of Lipid Transfer in
Microbubble-Mediated Drug Delivery. Langmuir. 35 (40), 13205-13215 (2019).

71. Kinsler, L.E., Frey, A.R., Coppens, A.B., Sanders, J.V. Fundamentals of Acoustics, 4th Edition. ISBN: 0-471-84789-5 Wiley. (2000).

72. Wear, K.A. Considerations for Choosing Sensitive Element Size for Needle and Fiber-Optic HydrophonesPart I: Spatiotemporal Transfer Function and Graphical Guide. IEEE Transactions on Ultrasonics, Ferroelectrics, and Frequency Control. 66 (2), 318-339 (2019).

73. Stoica, Petre, Moses, R. Spectral Analysis of Signals. Prentice Hall. Upper Saddle River, NJ. (2005).

74. Lyka, E., Coviello, C., Kozick, R., Coussios, C.C. Sum-of-harmonics method for improved narrowband and broadband signal quantification during passive monitoring of ultrasound therapies. Journal of the Acoustical Society of America. 140 (1), 741-754 (2016).

75. Barrack, T., Stride, E. Microbubble Destruction During Intravenous Administration: A Preliminary Study. Ultrasound in Medicine and Biology. 35 (3), 515-522 (2009).

76. Apfel, R.E. Acoustic cavitation. Methods in Experimental Physics. 19 (C), 355-411 (1981).

77. Mulvana, H., Stride, E., Tang, M.X., Hajnal, J.V., Eckersley, R.J. The Influence of Gas Saturation on Microbubble Stability. Ultrasound in Medicine and Biology. 38 (6), 1097-100 (2012).

78. Mulvana, H., Stride, E., Hajnal, J.V., Eckersley, R.J. Temperature dependent behavior of ultrasound contrast agents. Ultrasound in Medicine and Biology. 36 (6), 925-34 (2010). 
79. Mulvana, H., Eckersley, R.J., Tang, M.X., Pankhurst, Q., Stride, E. Theoretical and Experimental Characterisation of Magnetic Microbubbles. Ultrasound in Medicine and Biology. 38 (5), 864-75 (2012).

80. Coviello, C. et al. Passive acoustic mapping utilizing optimal beamforming in ultrasound therapy monitoring. Journal of the Acoustical Society of America. 137 (5), 2573-2585 (2015). 